\title{
Improving the Probing Depth of Thermographic Inspections of Polymer Composite Materials
}

\author{
G. Olafsson ${ }^{1}$, R. C. Tighe ${ }^{2}$, J. M. Dulieu-Barton ${ }^{1}$ \\ 1. University of Southampton, Faculty of Engineering and Environment, University Road, \\ Southampton, UK \\ 2. University of Waikato, Faculty of Science and Engineering, Private Bag 3105, Hamilton \\ 3240, New Zealand
}

\begin{abstract}
The maximum depth for which a defect of a given size can be detected in thermographic inspections is known as the 'probing depth'. For materials such as polymer composites, with low through-thickness thermal diffusivity, inspections are limited to thin materials or near surface inspection. With the aim of improving probing depth, the paper describes how established signal processing techniques are adapted for thermographic inspections. The procedures are implemented for both Pulse Thermography (PT) and Pulse Phase Thermography (PPT) inspections of laminated composite materials and sandwich structures. It is demonstrated that the adaptation significantly improves the probing depth, identifying defects that could not be identified using existing procedures. The applicability of the new approaches is discussed, paying particular attention to systematic and random errors resulting from equipment setup. Simple and efficient compensation methods to reduce the effect these errors are presented.
\end{abstract}

Keywords: Pulse Thermography (PT), Pulse Phase Thermography (PPT), Composite Materials, Image Processing.

\section{Introduction}

The use of composite materials has increased in a broad range of industries in recent decades; for example, as much as 50\% of modern aircraft like the Boeing 787 are now manufactured using composite materials [1]. While composite materials bring many advantages, enabling 
stiff lightweight structural design, there are also associated challenges. Defects in any material reduce mechanical performance and can lead to premature component failure. However, composite materials are particularly susceptible to manufacturing defects as the material and component are manufactured simultaneously [2]. Composite materials are also known for poor resistance to in-service events such as impact. This subject has been extensively studied, and it has been shown that even a low velocity impact can cause significant damage that is difficult to identify visually [3]. Therefore, alongside the increased use of composite materials there has also been an increased focus on non-destructive testing (NDT) of composites. As composite materials were initially implemented into structures and components, traditional NDT techniques such as ultrasound and radiography were used, many of which were originally developed to inspect metallic specimens [4]. While these techniques are still commonly used to this day, in recent decades thermographic NDT techniques have become popular for the inspection of composite materials [5]. Perhaps the most common of these is Pulse Thermography (PT), which has become an established NDT technique for the inspection of composite components, particularly for aerospace applications [6]. PT inspections use an infrared detector to provide full field measurement by monitoring the thermal decay of the surface of a component after pulse heating. Defects are identified by differences in thermal properties between defective and non-defective regions of components and structures. Most defects cause a local reduction in thermal diffusivity. When exposed to a heat pulse, this causes in an increase in the surface temperature of the component local to the defect, allowing defects to be identified using thermography and hence the nomenclature 'pulsed thermography or PT'.

More than two decades ago, Maldague and Marinetti [7] first proposed Pulse Phase Thermography (PPT), which introduced a post processing procedure, where the temperature data is transformed to the frequency domain to obtain phase data which is used to identify defects. The approach aimed to combine the simplicity and rapid inspection time associated with pulse thermography with a means to improve probe depth. In [7], PPT was shown to be less sensitive to surface effects such as on surface texture, variations in emissivity and nonuniform heating, all of which significantly influence PT inspections. Since PPT was initially proposed [7], the implementation of the technique has remained fundamentally unchanged, although a number of papers have developed the theory and expanded the technique to obtain additional information such as defect depth, e.g. [8]. However, frequency domain analysis of arbitrary signals is used in an enormous range signal processing approaches and is not unique to PPT. 
The purpose of this paper is to harness techniques from wider signal processing applications to develop a data processing procedure specifically to improve the probing depth of thermographic inspections of composite materials. Initially systematic and random errors present in the thermal data are discussed and a compensation strategy for these errors is presented. The compensated thermal data is then used with the PPT alongside the improved frequency domain analysis enabled by using advanced signal processing techniques.

\section{Pulse Thermography}

Pulse thermography exploits transient thermal regimes and is a type of active thermography, requiring external thermal excitation [6]. Figure 1 shows a typical inspection set-up for PT. A heat pulse is applied to the surface of a specimen, often using an optical flash that heats the specimen surface by radiation [9]. An infrared (IR) detector radiometrically measures the surface temperature evolution during cooling. PT can be carried out in transmission mode, where the heat source and IR detector are positioned opposite sides of a component. However, reflection mode, shown in Figure 1 is more commonly used as it allows single sided inspections with both heat source and IR detector positioned on the same side. In this configuration subsurface defects disturb the flow of heat through the material thickness, resulting in differences in surface temperature between defective and non-defective regions [10]. Defects are identified by comparing the temperature evolution measured at defective and non-defective regions within the field of view of an IR camera to produce a full-field image. Typical IR detectors used for PT and PPT are based on photon detectors [11] with focal plane arrays (FPA) of around $300 \times 300$ sensors and can record data at a frame rates up to $1 \mathrm{kHz}$. Meaning the image data is spatially rich, allowing visualisation of the defect, and temporally rich, allowing application of advanced signal processing procedures to extract frequency domain data. 


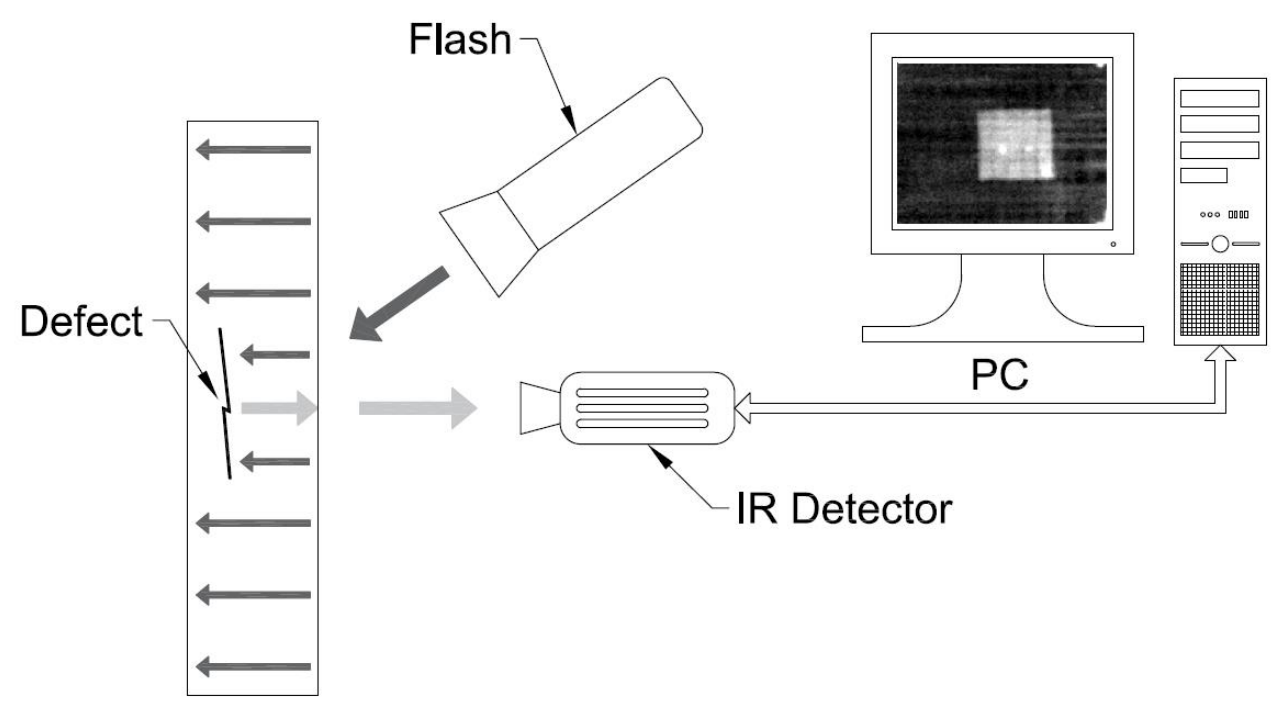

Figure 1: Experimental setup showing pulse thermography carried out in reflection mode

As with any measurement, the thermal data acquired by the IR camera system contains errors, due to inherent limitations of the experimental setup and equipment used. For example, FPA photon detectors must be cooled to operate. However, the reflection of the cooled sensor on the surface of the object being inspected can result in thermal non-uniformity, seen as a vignette effect, or cold spot in the centre of the field of view. In [12] a bi-hexic function was used to mathematically describe the effect of the cooled sensor. Using this function, the vignette effect can be subtracted from all frames of the thermal data set. In the present paper, a simpler method is adopted, where a reference image is generated by using thermal data recorded at ambient temperature prior to heating.

Another significant source of thermal non-uniformity is the positioning of the heat source relative to the inspected component. Ideally, the heat should be applied uniformly across the surface, but is practically impossible to achieve, not least because the heat source and IR camera cannot occupy the same space. Heat sources are therefore typically positioned to one side of the IR camera as shown in Figure 1. A way of reducing the non-uniform heating is to use two heat sources either side of the camera but this results in synchronisation issues and adds complexity to the equipment set up, which is not desirable for inspections in the field. In the present paper, a routine is developed that compensates for the thermal gradient caused by non-uniform heating in the thermal data for each image frame. 
In addition to thermal non-uniformity, which is a type of systematic error, the thermal data obtained from an IR detector will always contain random error. The dominant random error is temporal noise due to the analogue to digital conversion of the voltage output of the IR detector. This noise can adversely affect NDE inspections where the thermal contrast between defective and non-defective regions is low and the defects may be obscured by noise. A recent review by Vavilov and Burleigh [6] provided a high level overview of prominent strategies for temporal noise suppression. Thermal Signal Reconstruction (TSR) is one of the strategies discussed in [6] and has been highlighted by others [13,14] as being a particularly effective technique for temporal noise suppression. TSR was first proposed by Shepard et al. [15], and applies smoothing logarithmically rather than linearly. They assumed a 1D Fourier heat conduction model, where thermal decay for a homogeneous material was expressed as:

$$
\ln (T)=\ln \left(\frac{Q}{e}\right)-\frac{1}{2} \ln (\pi t)
$$

where $T$ is temperature, $Q$ is heat flux, $e$ is the thermal effusivity, and $t$ is time.

Equation (1) shows that by expressing the thermal decay in logarithmic terms, the decay becomes linear with a gradient of -0.5 . It is possible to exploit this when analysing experimental data containing high frequency temporal noise, by fitting a low order polynomial to the data. This acts as a low pass filter, smoothing out of non-physical responses associated with camera noise, by removing data that does not fit the assumed conduction model. By selecting an appropriately low polynomial order, typically a $6^{\text {th }}$ order as originally proposed in [15], it is possible to capture non-linear response at defective regions, while omitting high frequency noise.

\section{Pulse Phase Thermography}

The PPT technique exploits the fact that temporal signals can be approximated by a summation of a multitude of base sinusoidal waveforms of varying frequency, amplitude and phase. By transforming the temporal thermal decay signal measured at each pixel by the IR camera, to the frequency domain it is possible decompose the temporal signal into its constituent frequency components. This is commonly achieved using a Discrete Fourier Transform (DFT) [7], although the use of wavelet analysis has also been proposed [16]. For digital signals, the DFT is typically implemented using the Fast Fourier Transform (FFT) algorithm [17]. The FFT 
is simply an efficient implementation of the DFT developed for computational analysis of digital signals. The DFT is given by:

$$
F(n)=\sum_{x=0}^{N-1} f(x) e^{2 \pi i x n / N}=R e_{n}+i \operatorname{Im}_{n}
$$

where the temporal signal $f(x)$ is approximated in the frequency domain by $F(n)$, and $\mathrm{x}$ is a frame of the thermal data set, $N$ is the total number of frames, $n$ is the frequency increment. The phase of the response is given by:

$$
\phi=\tan ^{-1}\left(\frac{\operatorname{Im}}{R e}\right)
$$

where $R e$ and Im are the real and imaginary components of the complex number returned by the DFT. The magnitude of the response is given by:

$$
M=\sqrt{I m^{2}+R e^{2}}
$$

The DFT decomposes the temporal signal and sorts it by frequency, with data returned in frequency 'bins' numbered to $N$. The maximum frequency returned by the DFT is given by the Nyquist frequency $\left(F_{\text {nyquist }}\right)$ which is expressed as:

$$
F_{\text {nyquist }}=\frac{F_{s}}{2}
$$

where $\left(F_{S}\right)$ is the frame rate of the camera (i.e. the sampling rate) selected when acquiring thermal data.

The width of each frequency bin is given by the minimum frequency $\left(F_{\min }\right)$ :

$$
F_{\min }=\frac{F_{s}}{N}
$$

The frequency at each bin $(F(n))$ can be resolved using:

$$
F(n)=F_{\min } n
$$


The thermal decay of a defective region will differ from a non-defective region in the time domain and the same is true in the frequency domain. For each pixel, and each frequency the phase is calculated using equation (3), allowing the phase to be compared spatially in the image data.

Sorting data by frequency is advantageous since noise typically manifests as high frequency components of the temporal signal, while comparatively low frequencies describe thermal decay of the specimen surface. Separating low frequencies from high can allow for the identification of deep defects, which are otherwise obscured by detector noise in the thermal data. PPT can improve probe depth by increasing the contrast between defective and nondefective regions when compared to raw thermal data obtained for PT inspections. Similarly, phase data is less sensitive to surface effects such as non-uniform emissivity, and surface geometry is reduced when compared to PT thermal data. In this way, PPT is very similar to Lock-In Thermography (LT) [18], which also utilises phase data rather than raw thermal data. LT uses harmonic thermal excitation and a lock in algorithm to obtain the phase of the response at each pixel. Unfortunately, the frequency must be chosen prior to data acquisition, and if another frequency is to be considered, the inspection must be repeated. A key advantage of PPT is that a single inspection can be post processed to consider a multitude of frequencies.

Ibarra-Castanedo et al. [13] noted that noise in the thermal data can also affect phase data in PPT inspections, particularly at high frequencies. The study proposed the combination of TSR and PPT and provided an example using an aircraft rudder. A further study by Ibarra-Castanedo and Maldague [19] demonstrated the effect of TSR on PPT phase data, comparing phase data obtained from TSR and unsmoothed temporal thermal data. However, while significant improvements were reported, TSR has not been widely implemented in the general PPT literature.

Ibarra-Castanedo and Maldague [20] outlined an optimised procedure for PPT. Sampling rate and acquisition time are selected based on the thermal properties of the inspected material. Lower frequencies are of interest for increasingly deep defects. It is therefore often advantageous to minimise the width of frequency bins. As can be seen from equation (6), this can be achieved by either increasing the number of samples by increasing the recording duration, or by decreasing the recording frame rate. However, the number of samples acquired over a given duration is linearly proportional to the sampling rate. Therefore, to reduce the minimum frequency resolved, the duration of data acquisition must be increased. Clearly, there 
is a practical limitation, as the specimen surface will eventually return to ambient temperature. Furthermore, as the maximum resolvable frequency is limited by the Nyquist frequency, the sampling frequency must be sufficiently high to ensure that all frequencies of interest are captured.

Once data from PT inspections is acquired, the thermal response measured at each pixel is digitally recorded as a transient signal. Ibarra Castenado and Maldague [20] proposed the truncation of the signals, by applying a rectangular window, disregarding a number of initial data points. A rectangular window gives a weighting of one for all values sampled and zero for all other values, and its length is chosen to maximise the number of samples, minimising the frequency bin width using an iterative procedure. By making the window size smaller than the original signal, data is excluded from the start of the signal, which improves the contrast. Although, in [20] it is not explained why this is effective, a possible explanation is that removing the first part of the response makes the resulting signals less transient (or more stationary) and better suited for analysis with DFT. In a more recent paper, Ibarra-Castanedo and Maldague [19] again suggest the use of a rectangular window, and revisit the implementation of PPT. However, little discussion is provided to justify the choice of the rectangular window to apply the DFT. Rectangular windows only work well when the frequencies of interest are known so a judicious choice of sampling parameters can be made [21]. Specifically, a non-zero and exact integer number of cycles of a waveform must be sampled, otherwise periodic extension of the sampled waveform results in discontinuities in the temporal signal. This results in a distribution of spectral energy from one frequency to all other frequencies considered in the DFT known as spectral leakage [22]. Often in PPT, many frequencies are of interest and these are not known prior to data acquisition, making it impossible to sample to avoid spectral leakage. Consequently, in PPT there will always be spectral leakage if a rectangular window is used and by definition probing depth is restricted. To the authors' knowledge, the implications using different sampling window configurations for PPT has not been previously studied. In the present paper, three windows are compared with the aim of understanding how spectral leakage affects defect identification in PPT. It is shown that using more effective windows for the DFT provides a marked improvement on defect identification, particularly when combined with TSR and initial image processing of the thermal data. 


\section{Considerations for thermal data processing for PPT}

A common strategy [22] for mitigating the effects of spectral leakage is to apply a weighting to the temporal signal, i.e. a windowing function. The rectangular window is attractive due to its simplicity as it provides a narrow main lobe in the frequency domain, and therefore high frequency resolution. However this is at the cost of spectral leakage [22], which can be seen as large side lobes in Figure 2 where the frequency domain representation of an exponential signal similar to thermal signals from PT is shown. Most windowing functions aim to reduce spectral leakage by reducing the discontinuity occurring in waveforms that are not periodic in the observation window. This is achieved by weighting the input temporal signal such that the signal ends approach zero smoothly. Ultimately, there is always a trade-off between spectral leakage and frequency resolution as shown in Figure 2, where the flat top window almost eliminates leakage, but results in a wide main lobe.

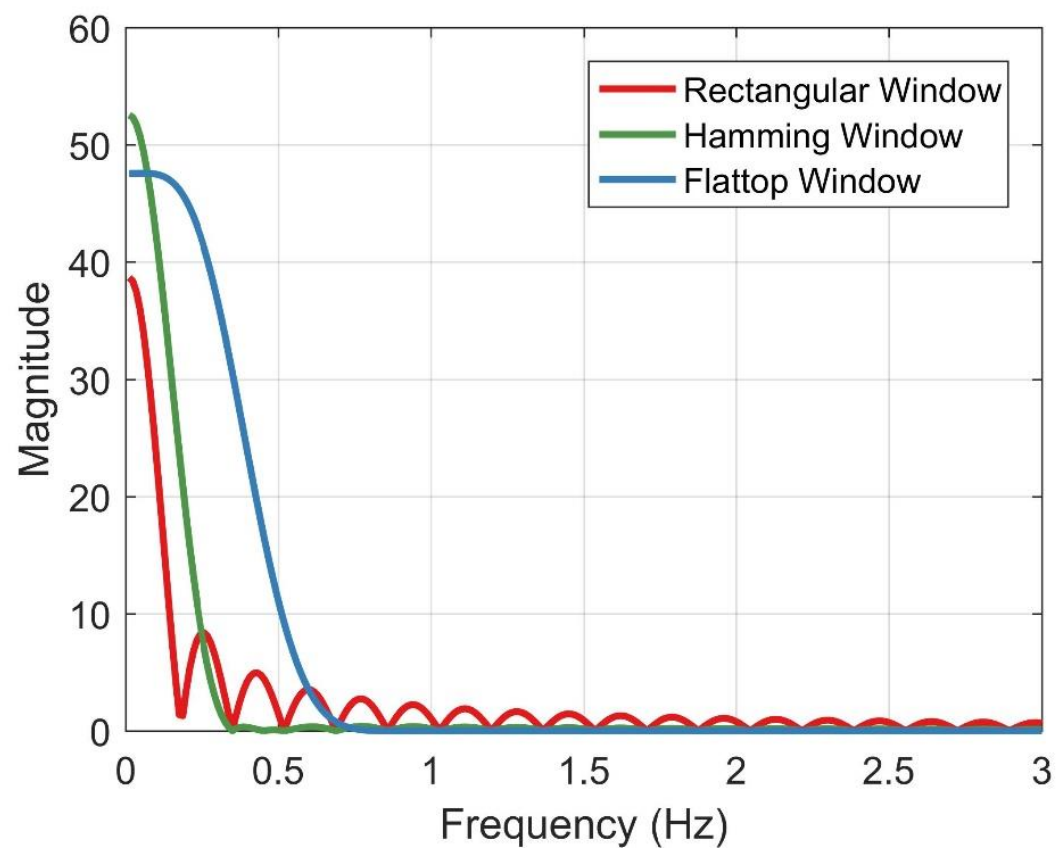

Figure 2: Frequency response of windowed exponential signal

In view of the discussion above, three windowing functions (rectangular, hamming and flattop) are investigated for PPT. The rectangular window function is:
$w(n)=1$
$|n| \leq N-1$
$=0$
otherwise 
Olafsson et al.2018 Measurement Science \& Technology

where $w(n)$ denotes the windowing function.

The hamming function is based on a cosine waveform, which approaches zero and hence reduces spectral leakage by reducing the discontinuity of constituent sinusoids. By not crossing zero, the hamming functions also result in a modest increase in main lobe width. The hamming function is as follows:

$$
\begin{aligned}
w(n) & =0.54-0.46 \sin \left(\frac{2 \pi n}{N-1}\right) & & |n| \leq N-1 \\
& =0 & & \text { otherwise }
\end{aligned}
$$

The flattop window is based on the sum of cosine waveforms and crosses zero, which results in low spectral leakage at the cost of main lobe width. The flatop window is of interest here because PPT is a comparative technique, and the increase in main lobe width applies to all thermal signals in a data set. Therefore the flat top window presents an opportunity to study the sensitivity of the data to main lobe width increase and side lobe attenuation. The flattop window function is:

$$
\begin{aligned}
& w(n)=a_{0}+a_{1} \cos \left(\frac{2 \pi n}{N-1}\right)+a_{2}+\cos \left(\frac{4 \pi n}{N-1}\right)+a_{3} \cos \left(\frac{6 \pi n}{N-1}\right)+a_{4} \cos \left(\frac{8 \pi n}{N-1}\right) \\
& \text { where } 0 \leq n \leq N-1
\end{aligned}
$$

where $a_{0}-a_{4}$ are coefficients, the values used are presented in Table 1. 
Olafsson et al.2018 Measurement Science \& Technology

Table 1: Coefficients for Flattop Window Function

\begin{tabular}{cl}
\hline Coefficient & Value \\
\hline$a_{0}$ & 0.21557895 \\
$a_{1}$ & 0.41663158 \\
$a_{2}$ & 0.277263158 \\
$a_{3}$ & 0.083578947 \\
$a_{4}$ & 0.006947368 \\
\hline
\end{tabular}

A further consideration is the minimum resolvable frequency determined by the sampling frequency and the number of samples [22]. This can only be improved by increasing recording duration, which is limited by the transient thermal response. Therefore zero-padding [23] is used, which increases the number of samples and reduces the minimum frequency bin width. Zero-padding does not however provide additional signal information, and can therefore be considered analogous to interpolating between bins. This approach has not be widely applied in PPT studies to date, with very few authors reporting its use. Weritz et al. [24] reports the use of zero-padding but there is no comparison with non-padded data, hence in the present paper the effect of zero-padding of signals for PPT is evaluated, alongside the effect of using different windows in the DFT. 


\section{Experimental Setup}

\subsection{Materials and Manufacturing}

A common defect in composite sandwich panels is delamination, which occurs at the interfaces of the plies in the face sheet laminate, as well as at the interface between the core and the face sheet. A thermographic technique that could identify defects in sandwich structures would be attractive as it is fast and non-contact and could be used as part of in-service inspections as well as following manufacture. Therefore, a test component comprising a composite sandwich panel with glass reinforced fibre epoxy face sheets and a foam core was manufactured in a cocure resin infusion process. Interfacial defects were simulated by placing $20 \mathrm{x} 20 \mathrm{~mm}$ Polytetrafluoroethylene (PTFE) inserts between each ply of the laminate. Figure 3 shows a schematic of the test panel with relevant dimensions. The sandwich panel face sheet is shown as an exploded view so the position of the PTFE patches through the thickness of the face sheet are easily represented. The specimen was designed such that the limits of probing depth could be established, involving a combination of shallow and deep defects. The largest defect depth was chosen so that it could not be detected using PT alone without the additional processing described in the present paper. The specification of materials used for the face sheets and core are presented in Table 2 . 
Olafsson et al.2018 Measurement Science \& Technology

Table 2: Material Specification

\begin{tabular}{lll}
\hline Panel Part & Description & Specification \\
\hline Core & Type & Closed Cell PVC \\
& Manufacturer & Diab \\
& Name & Divinycell H250 \\
& Density & $250 \mathrm{~kg} / \mathrm{m}^{3}$ \\
& Thickness & $35 \mathrm{~mm}$ \\
& Thermal Conductivity & $0.049 \mathrm{~W} / \mathrm{m} . \mathrm{K}$ \\
\hline Face Sheet & Type & Glass Fibre Reinforced Polymer \\
& Reinforcement Type & Biaxial Stitched E-Glass \\
& Number of Plies & 4 \\
& Ply Thickness & $0.6 \mathrm{~mm}$ \\
& Laminate Thickness & $2.4 \mathrm{~mm}$ \\
Reinforcement Specification & $570 \mathrm{~g} / \mathrm{m}^{2}$ \\
Resin Type & Epoxy \\
Resin System & Gurit Prime 20 LV \\
Resin Hardener & Gurit Prime 20 Fast Hardener \\
\hline
\end{tabular}


Olafsson et al.2018 Measurement Science \& Technology

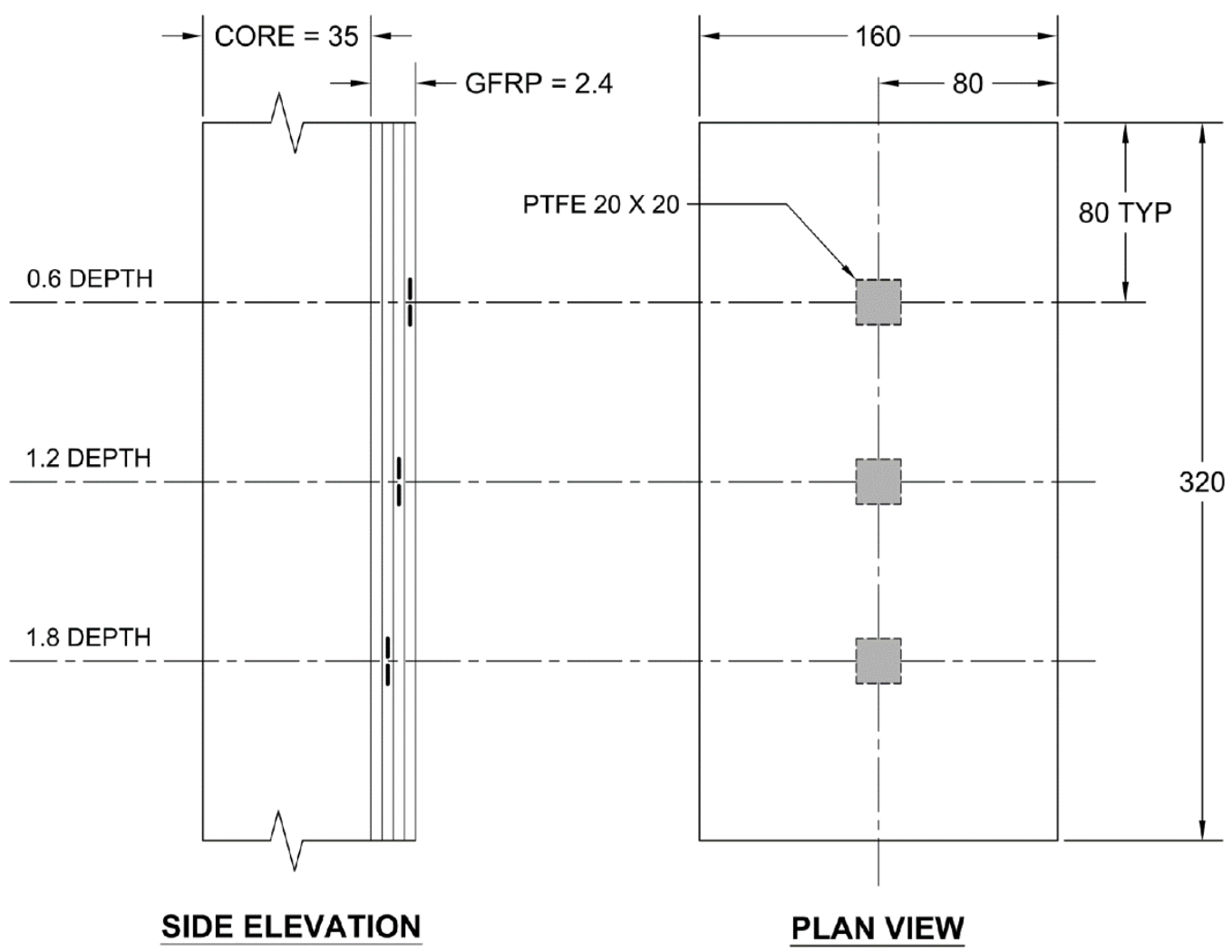

Figure 3: Schematic showing specimen configuration (not to scale - all dimensions are in $\mathrm{mm}$ ) 


\subsection{Experimental Methodology}

Pulse thermography was used to obtain all data presented in this study. The IR detector stand off was selected to ensure both defective and non-defective regions were clearly within the field of view. A photographic flash was used to apply a short duration (pulse) thermal excitation to the surface of the specimen. This was placed as close to the specimen as possible to maximise the heat input to the specimen and hence the thermal contrast obtained. The IR detector was perpendicular to the component surface, and the heat source positioned at angle. This reduced perspective effects, and as will be shown, the thermal non-uniformity that resulted is predictable and relatively simple to compensate. The subsequent thermal decay was measured using an IR detector. Pulse thermography was carried out in reflection mode as described in [25], and shown in Figure 1. It is important to note that the surface of the specimen was not painted with matt black paint. The epoxy resin surface of the GFRP face sheet material had a high emissivity and it was found that the paint insulated the surface and decreased thermal contrast, which discounted any improvements in surface emissivity. The specification of the IR detector and flash are provided in Table 3.

Table 3: Equipment Specification

\begin{tabular}{lll}
\hline Equipment & Description & Specification \\
\hline Photon Detector & Model & Cedip Silver 480m \\
& Thermal Sensitivity & $20 \mathrm{mK}$ \\
& Sensor & $\mathrm{InSb} \mathrm{320} \mathrm{x} \mathrm{256} \mathrm{px} \mathrm{cooled} \mathrm{FPA}$ \\
& Spectral Range & $3.6-5.6 \mu \mathrm{m}$ \\
& Recording Frame Rate & $383 \mathrm{~Hz}$ \\
& Recording Duration & $6 \mathrm{~s}(2298$ frames $)$ \\
\hline Photographic Flash & Model & Bowens 1000 Pro \\
& Power & $1000 \mathrm{Ws}$ \\
& Flash Duration at Full Power & $1 / 2100 \mathrm{~s}$ \\
\hline
\end{tabular}

CIRRUS software was used for the IR detector control and initial setup, including the nonuniformity correction to adjust for variation in sensor sensitivity. Thermal data was captured using Altair provided by FLIR Systems. All of the thermal data was processed using a specially developed Matlab R2016 script. 


\section{Thermal Data Processing}

\subsection{Thermal Non-Uniformity}

Thermal non-uniformity over the field of view of the camera can obscure the temperature difference that distinguishing the defective from non-defective regions. The thermal nonuniformity may have larger temperature range across the field of view, which masks the visualisation of small temperature changes such as those resulting from deep sub surface defects. In this section processing procedures are presented to reduce and/or eliminate thermal non-uniformity and hence increase the depth at which the defects can be visualised. To evaluate the processing procedures, a thermal image series was captured from a region surrounding the PTFE insert placed at $0.6 \mathrm{~mm}$ depth from the surface of the sandwich structure component. The $0.6 \mathrm{~mm}$ defect depth was selected because the defect was identifiable in the raw data and provided a basis for comparison. Therefore the SNR could be calculated prior to processing being applied, so that an evaluation could be made of the improvements achieved by each procedure.

Figure 4 shows the thermal data obtained from the specimen at ambient temperature, which reveals a major source of non-uniformity in the thermal data caused by the reflection of the cooled IR detector. A vignette effect is produced with reduced temperature at the centre of the field of view. The vignette effect is present in all image frames in the thermal data set, but after pulse heating other effects are also visible in the thermal data and therefore is best visualised prior to heating. The vignette effect will vary slightly through the duration of the inspection, since the difference in temperature between the surface and sensor increases during heating, which is greatest in images captured immediately after heating. However, it was observed that this variation was generally small as the surface temperature quickly returns to near ambient, so it was assumed that the vignette was constant throughout the dataset. Hence, it is possible to capture a 'reference image' prior to heating which can then be subtracted from the remaining images in the series. To reduce the spatial noise, the reference image was produced from the temporal average of twenty frames captured before heating. The mean temperature of the reference image was then added to all the image frames as an offset to maintain actual temperature measurements. 


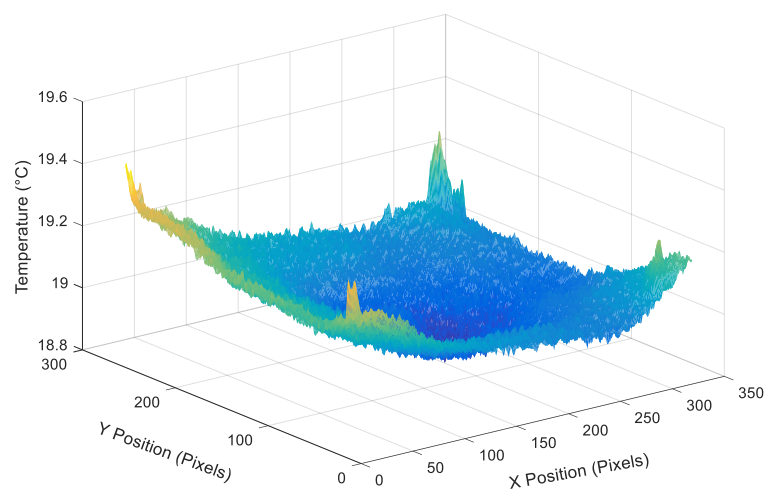

a) Surface plot

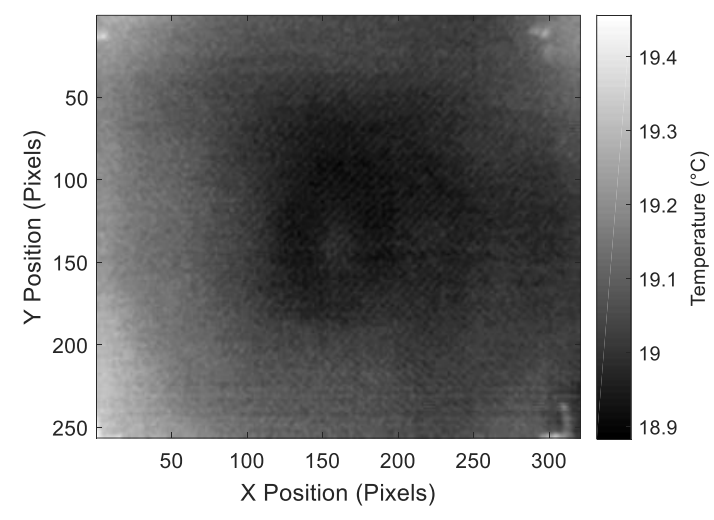

b) Thermal image

Figure 4: Thermal data measured prior to flash heating, showing vignette effect due to cooled IR detector sensor.

Subtracting the reference image almost completely eliminates the vignette as shown in Figure 5. However, a thermal gradient is clearly visible across the field of view, which is a result of non-uniform pulse heating. Since one side of the field of view is cooler than the other, the rate of cooling also varies across each frame and through time, so subtraction of a single reference frame cannot be used. Therefore compensation must take place frame by frame. This was achieved by averaging five rows of thermal data from the non-defective region and applying a first order polynomial fit to approximate the thermal gradient across the field of view for each frame. Once the gradient is known, it can be subtracted from all rows of a frame in the image series. The resulting data is presented in Figure 6, showing that the temperature gradient across the field of view is practically zero. In section 7.4 of the paper, it is shown that combining the thermal non-uniformity corrections with other processing procedures helps increase the probing depth. 


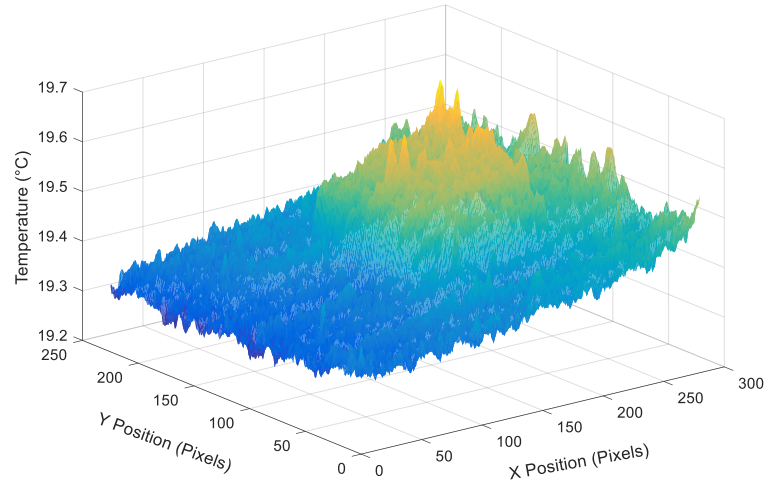

a) Surface plot

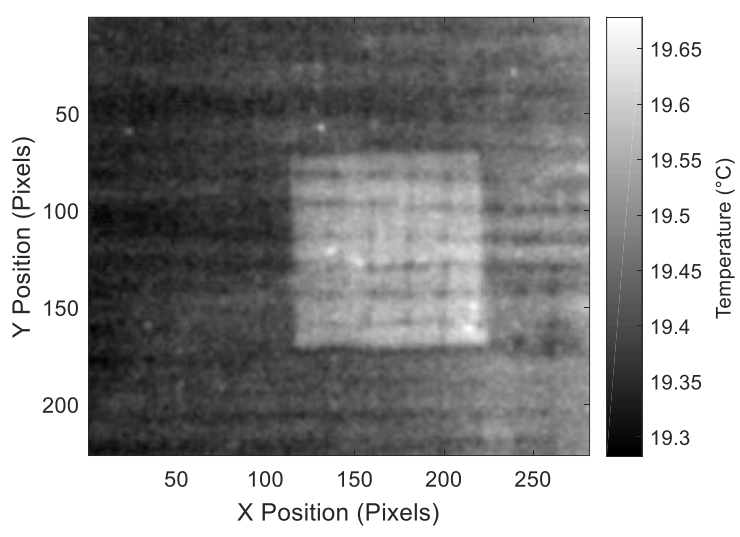

b) Thermal Image

Figure 5: Reference image subtracted, data at $\mathrm{t}=0.0757 \mathrm{~s}$ after flash heating.

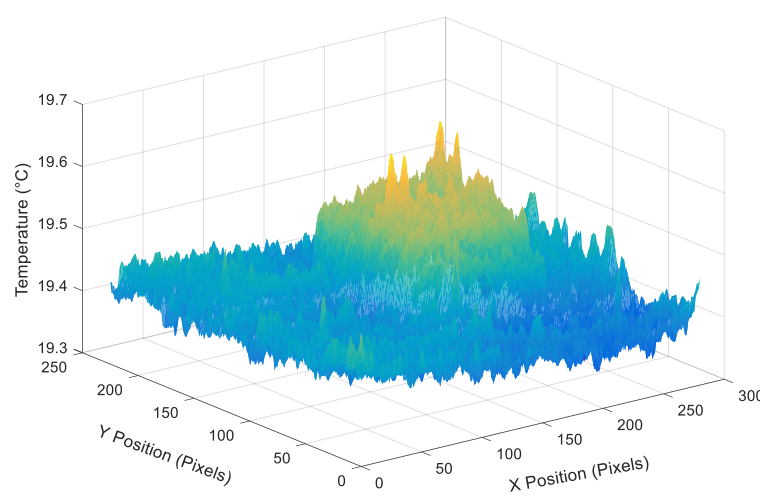

a) Surface Plot

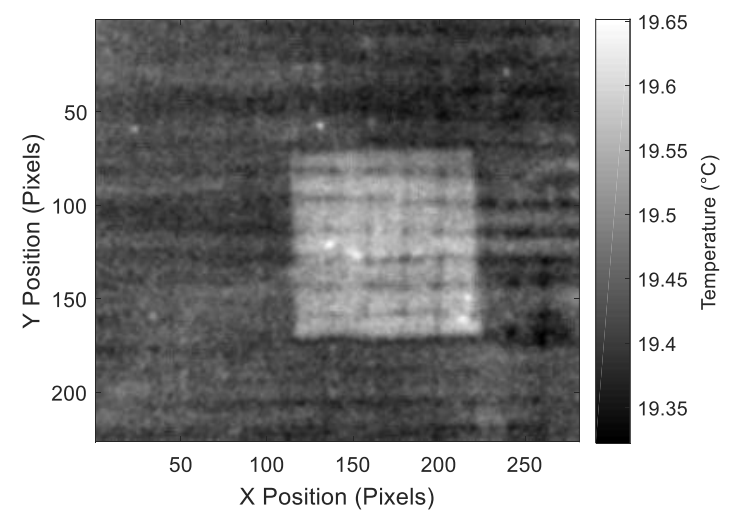

b) Thermal image

Figure 6: Reference frame subtracted, and compensated for flash, data at $\mathrm{t}=0.0757 \mathrm{~s}$ after heating.

\subsection{Temporal Noise}

Significant temporal noise can be observed in the acquired thermal data, as shown in Figure 7, which plots the temperature evolution of a single pixel in the image data in a non-defective region. Where thermal contrast is low, temporal noise can be sufficient to obscure small temperature variations thus restricting probing depth and as will be shown, temporal noise further restricts probing depth in the frequency domain. TSR [15] was used to smooth temporal 
noise and produce synthetic reconstructed images, as shown in Figure 7, where the noise reduction is clear while the thermal decay is preserved.

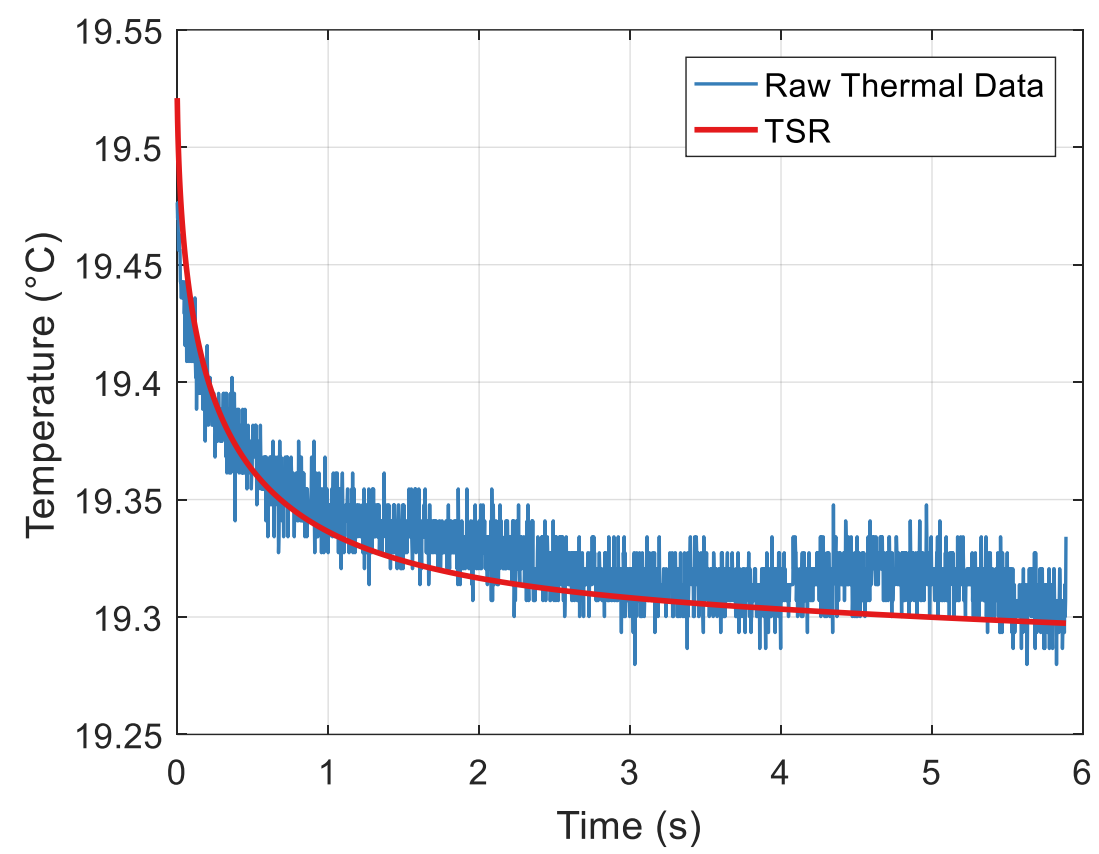

Figure 7: Raw data and TSR temperature evolution of a single pixel of a non-defective region.

\subsection{Improvements in probing depth resulting from the thermal processing}

Each processing technique was applied successively to the data obtained from the region including the shallowest defect. A reference image was subtracted from the raw data, flash compensation applied to the resulting data and finally TSR applied to the flash compensated data. The defective region was excluded and the $2 \mathrm{D}$ standard deviation of the response was calculated for each frame of the data set. The 2D standard deviation over the entire nondefective region in the image is presented in Figure 8 for each successive processing step. The raw data is not shown in Figure 8 since it was an order of magnitude higher than all the processed data. 


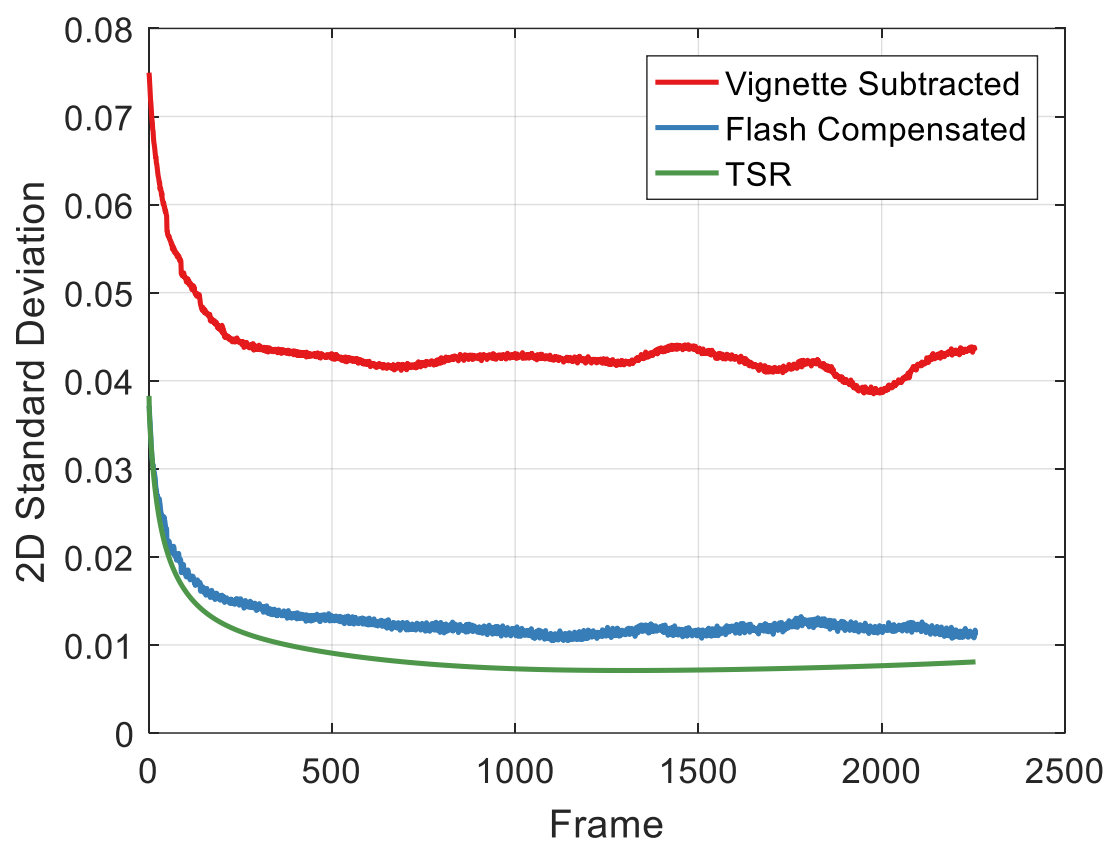

Figure 8: Comparison of 2D standard deviation of non-defective region after each processing technique is applied.

To further quantify the effect of these processing steps the signal noise ratio (SNR) was calculated as proposed by Vavilov [26] as:

$$
S N R=\frac{\overline{T_{d}}-\overline{T_{n d}}}{\sigma_{n d}}
$$

where $\overline{T_{d}}$ and $\overline{T_{n d}}$ is the average temperature in a defective region and non-defective regions respectively and $\sigma_{n d}$ is the 2D standard deviation of the non-defective regions. It is should be noted that the regions are manually selected after data acquisition.

Although the PTFE insert is visible in the raw data, the effect of thermal non-uniformity strongly influences the results. Figure 9 shows each successive processing procedure improves the SNR, indicating that the simulated defect is more clearly identifiable with each processing step. In addition to improving peak SNR, the processing also results in higher contrast for longer, which also improves identification. 


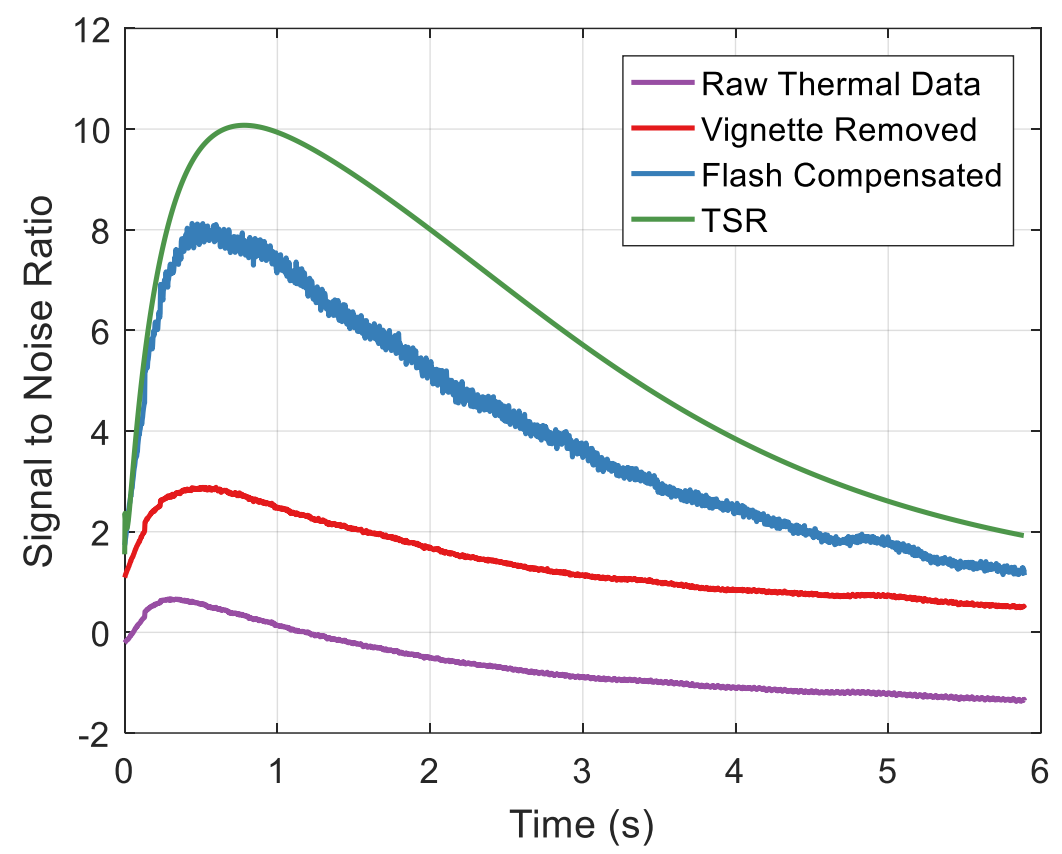

Figure 9: Signal to noise ratio calculated for each frame of the data set and for each processing step

To visually evaluate the effect of each step in the processing procedure, Figure 10 presents data from the area surrounding a PTFE insert at $1.2 \mathrm{~mm}$ depth. In the raw thermal data in Figure 10 (a) the PTFE is barely visible in the centre of the field of view. In Figure 10 (b) the vignette effect has been compensated for, the result is significantly improved and the PTFE insert is more clearly visible. However, significant non-uniformity remains due to the position of the flash during heating, resulting in an excessively large temperature range across the field of view. Figure 10 (c) shows the data after flash compensation resulting reduced non-uniformity, however spatial noise can be observed, which is due to the temporal noise in the data. When the temporal noise is compensated for using TSR as shown in Figure 10 (d), the spatial noise is reduced, and the simulated defect is clearly identifiable. In assessing the steps in the processing and the marked improvements at each step it is important to consider that in these trials the shape and location of the defect is known. In actual inspections the position of the defects are unknown and may be at the edges of the images, where the effect of the flash and the vignetting is more pronounced. The procedure described above enables all of the image to be used, allowing larger areas to be inspected rapidly and consistently. Furthermore, it would be necessary to apply all the processing steps to reveal deep defects of unknown location and shape. 


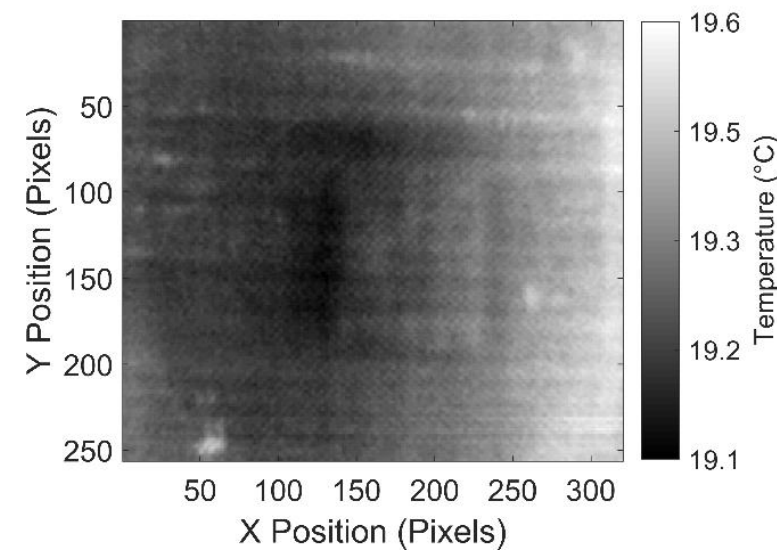

a) Raw thermal data

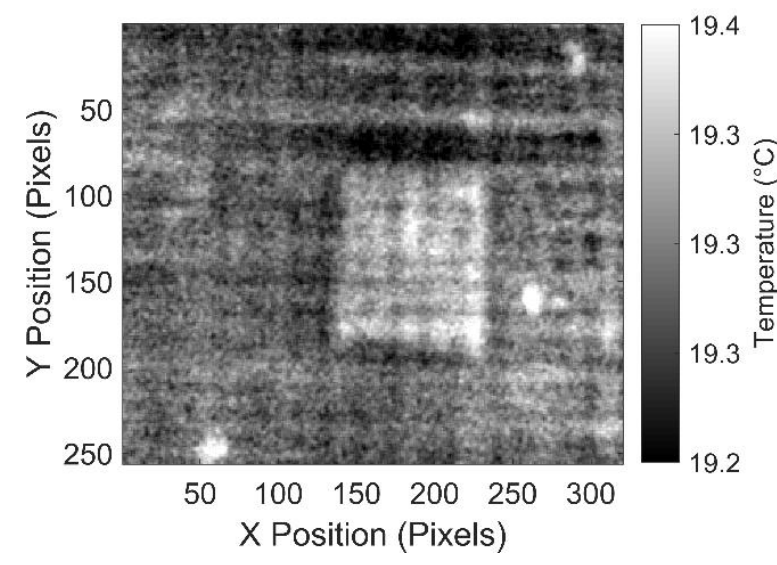

c) Flash compensated

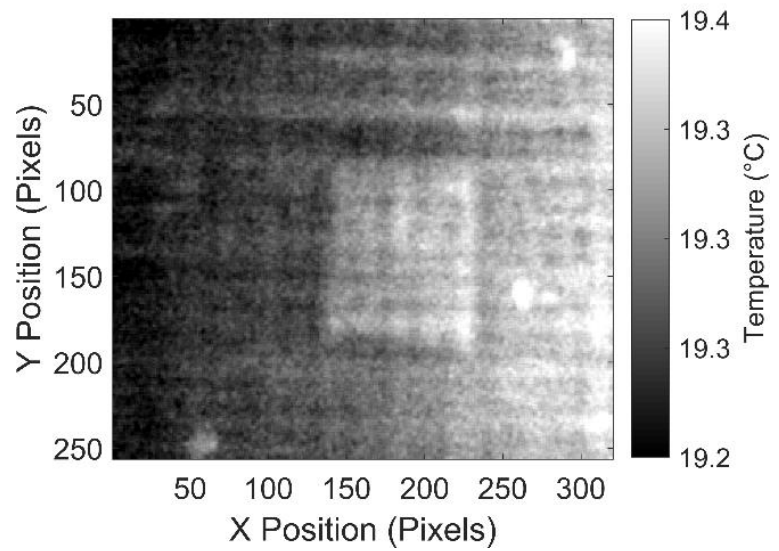

b) Vignette compensated

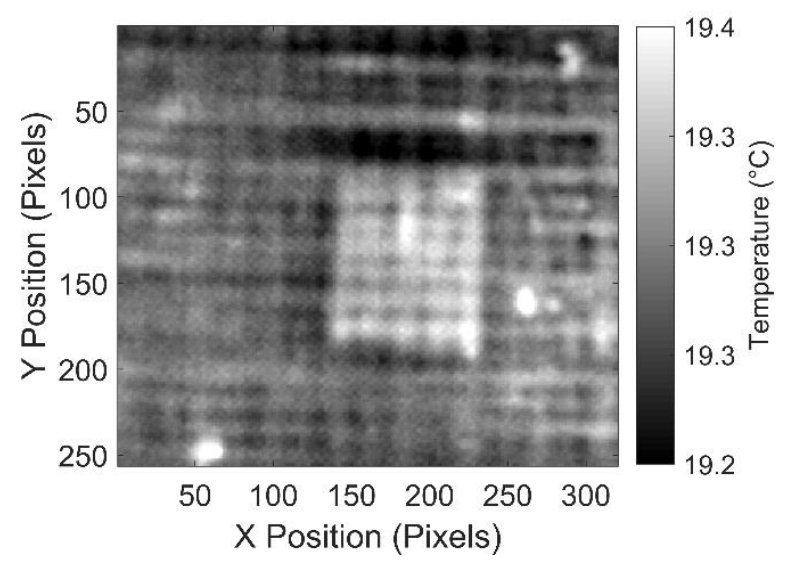

d) TSR data

Figure 10: Effect of errors in thermal data from inspection of PTFE insert at $1.2 \mathrm{~mm}$ depth at $\mathrm{t}=$ $0.6527 \mathrm{~s}$

\section{Processing for Pulse Phase Thermography}

\subsection{Temporal Smoothing}

The effect of combining TSR and PPT is shown in Figure 11 for a PTFE insert located at a depth of $0.6 \mathrm{~mm}$. The data was processed using a rectangular window function and $\mathrm{N}=2257$ samples. Figure 11 (a) shows the phase data obtained using raw thermal data. Figure 11 (b) shows the phase data when TSR was applied for temporal smoothing with a $6^{\text {th }}$ order polynomial. Noise in the phase data increases with increased frequency, since camera noise typically manifests as high frequencies $(>1 \mathrm{~Hz})$. These frequencies are therefore present in the input signal to the DFT, and are indistinguishable from the frequencies that result from the thermal decay of the specimen surface. This can be seen in Figure 11 (a), where at $4.1 \mathrm{~Hz}$, the 
simulated defect in the centre of the field of view is poorly characterised. When temporal smoothing is applied to the time domain signal, the input to the DFT no longer contains significant frequencies attributable to camera noise. Therefore, although TSR is a form of temporal smoothing, it significantly reduces the spatial noise in phase images, as shown in Figure 11 (b). At low frequencies $(<1 \mathrm{~Hz})$ noise suppression with TSR has a reduced effect in the frequency domain, as the camera noise is less in this frequency range.

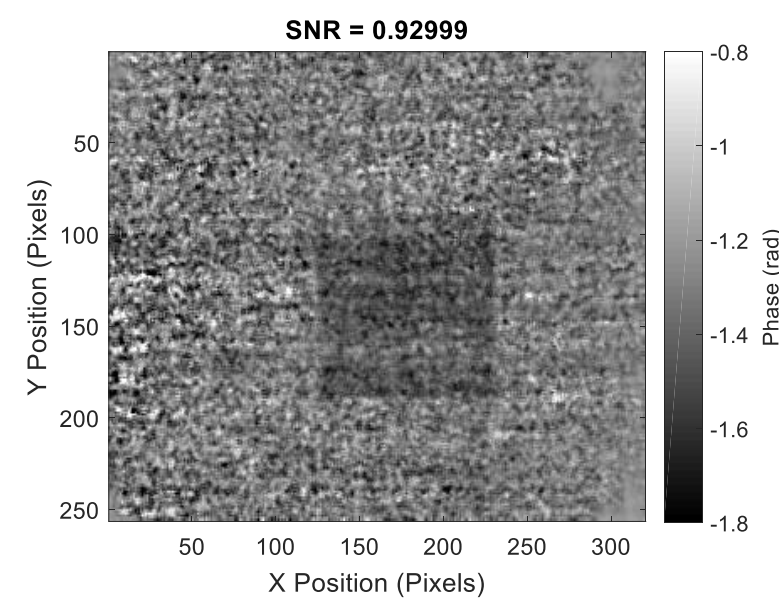

a) No temporal smoothing

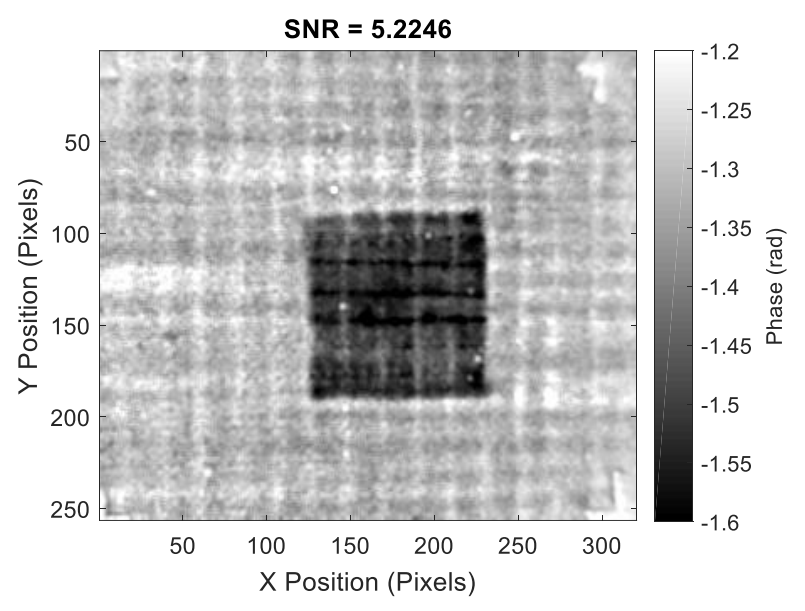

b) TSR applied to thermal data prior to PPT.

Figure 11: Effect of temporal smoothing using TSR on phase data for PTFE insert at 0.6 mm depth. Phase images at $4.1 \mathrm{~Hz}$.

Another interesting consequence of these observations is in the determination of blind frequency, which can be used for defect depth estimation. Blind frequency [8] is defined as the frequency at which the phase contrast between defective and non-defective regions reaches zero. Since higher frequency thermal waves are attenuated more easily, the frequency at which a defect is no longer identifiable can give an indication of defect depth. However, as shown in Figure 11, temporal noise in input signals to PPT results in phase noise, reducing phase contrast. Therefore, the blind frequency obtained using a smoothed temporal signal compared to raw data will differ, indicating that the blind frequency is dependent on the noise floor of the IR detector and hence the system being used for PPT. Using the same data and processing described in Figure 11, the phase image SNR was calculated for each frequency and is shown in Figure 12, comparing the results obtained using raw thermal data against TSR data. Using raw data, the SNR drops to close to zero at approximately $6 \mathrm{~Hz}$, where the SNR is 0.19 , and qualitatively there is no contrast between defective and non-defective regions as shown in 
Figure 13 (a). Although there are small variations, the phase contrast remains close to zero for frequencies greater than $6 \mathrm{~Hz}$. When TSR data is used, at $6 \mathrm{~Hz}$ the SNR is 2.9 and the PTFE insert is clearly visible in Figure 13 (b). The SNR when TSR is applied approaches zero at 10 Hz. The unsmoothed data at $10 \mathrm{~Hz}$ is shown in Figure 11 (c) is clearly zero, however in the TSR data of Figure 13 (d), the presence of the PTFE insert is apparent, albeit with low contrast. In fact by using TSR, the PTFE insert can be identified at almost all frequencies, and the SNR actually improves at frequencies in excess of $32 \mathrm{~Hz}$. This is likely due to the location of PTFE insert, close to the surface of the component face sheet. However, the data shown in these figures highlights the challenges associated with estimating blind frequency, and suggests that some form of temporal smoothing, such as TSR, could improve blind frequency estimation accuracy.

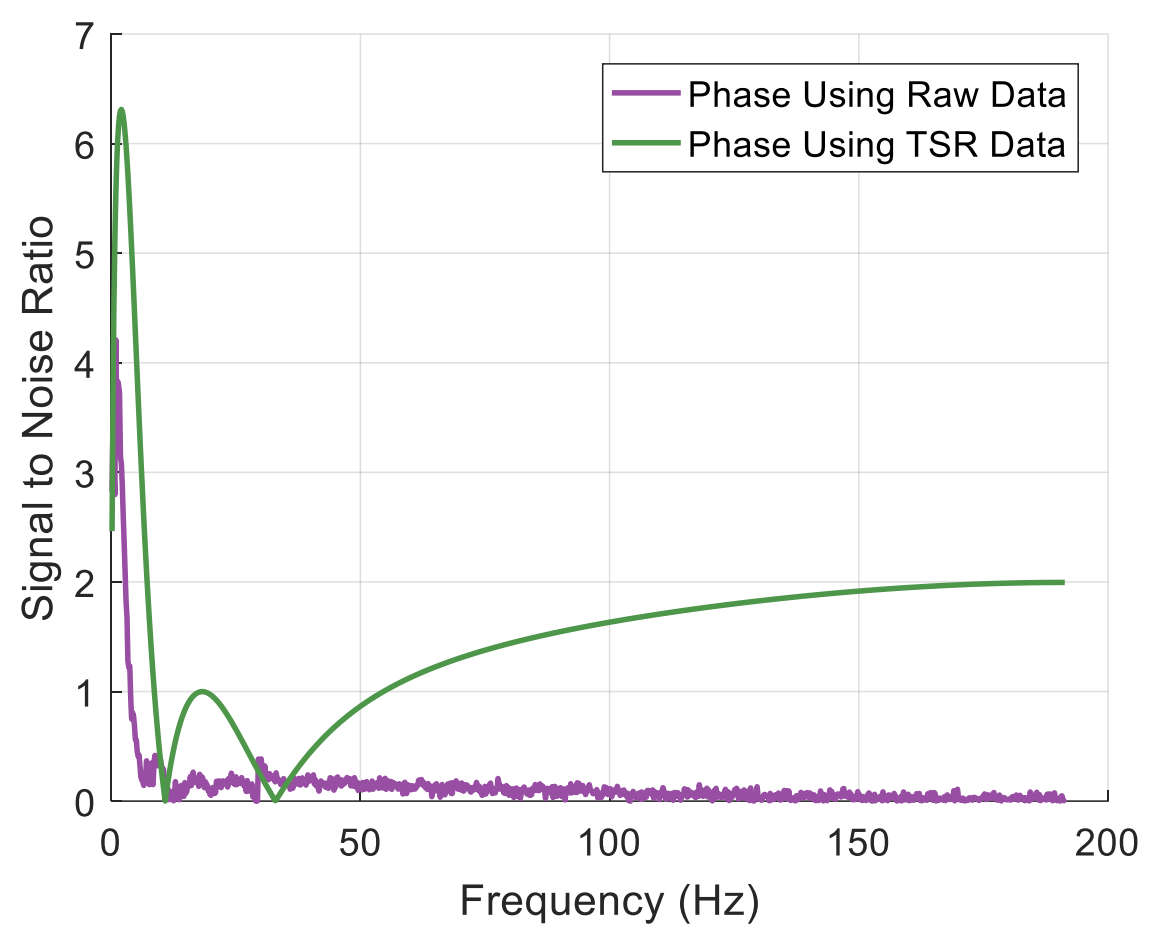

Figure 12: SNR with increasing frequency for phase data of PTFE insert at $0.6 \mathrm{~mm}$ depth. 


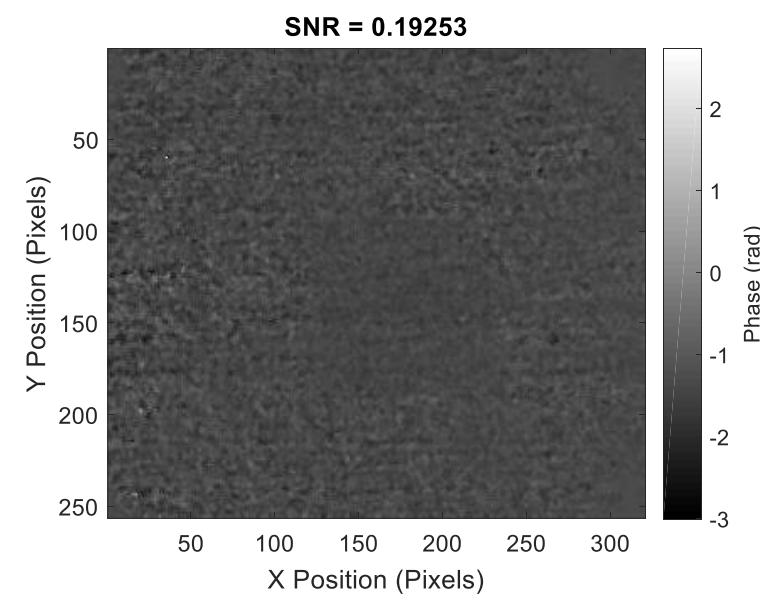

a) Unsmoothed $6 \mathrm{~Hz}$

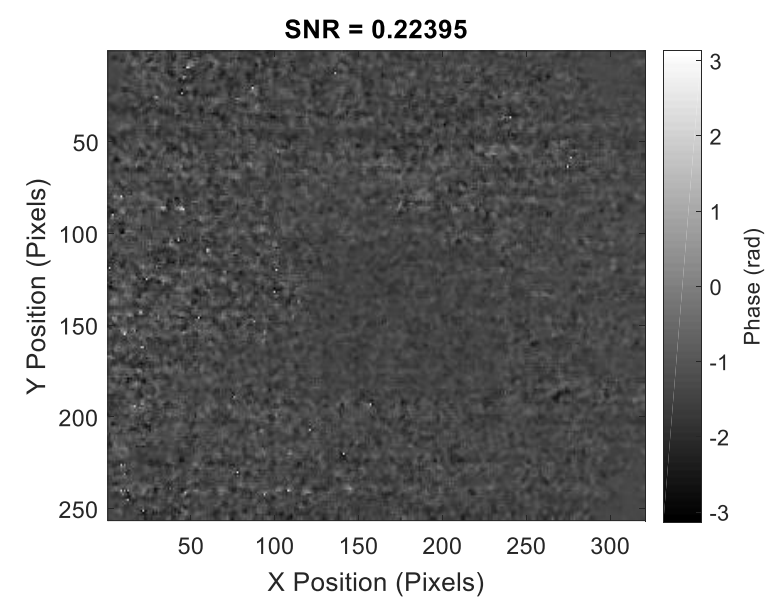

c) Unsmoothed $10 \mathrm{~Hz}$

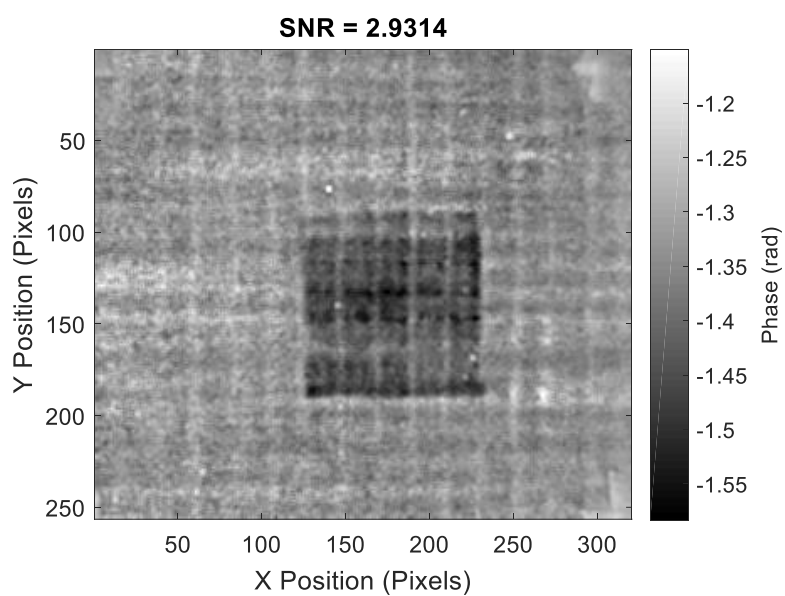

b) Smoothed $6 \mathrm{~Hz}$

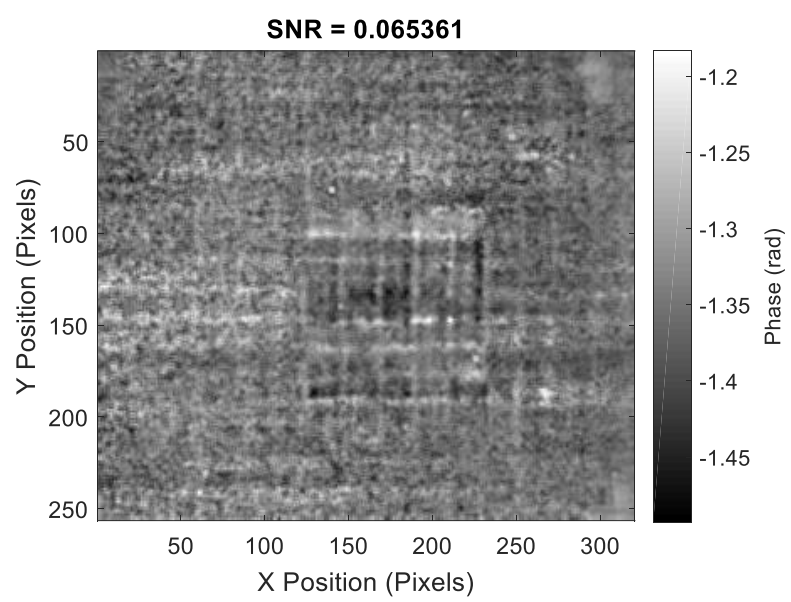

d) Smoothed $10 \mathrm{~Hz}$

Figure 13: Phase images comparing phase images obtained from PPT using raw thermal data and TSR data.

\subsection{Effect of Window}

Three window functions were compared using the same experimentally obtained thermal data. The phase data presented in Figure 14 shows a PTFE insert placed at $1.8 \mathrm{~mm}$ depth (between the third and fourth plies) in the sandwich panel face sheet. To highlight the effect of varying only the window function, raw thermal data, truncated to remove pre-flash frames, was used. The phase images corresponding to the highest SNR for each window are presented in Figure 14. It should be noted that with increasing frequency beyond the blind frequency, the PTFE insert is not visible in the phase images, so SNR is no longer valid as there is no phase contrast. Peak SNR images were identified using an automated routine in Matlab, and these were 
manually screened to confirm the PTFE insert was visible in the data. Using a rectangular window the SNR is high as shown in Figure 14 (a) however, the defect identification is poor. In addition, an inspection of several of the initial phase images showed similar phase distributions to Figure 14 (a), suggesting that the low frequency high, spectral energy, from the defective regions has leaked into other (higher) frequency bins. By contrast, the hamming window (Figure 14 (b)) results in a lower SNR but provides better defect identification, with the square shape of the PTFE clearly distinguishable and shows a marked improvement in identification over the rectangular window. The flattop window (Figure 14 (c)) provides the lowest contrast in this instance, with the PTFE insert is barely identifiable in only in the frequency bin shown in the image. However this could be because too few samples are used $(N=2048)$, resulting in wide frequency bins.

Spatial noise is present in all the phase data, because the raw thermal data is used without corrections. For example in Figure 14 (b), the top left of the image results in greater phase than the top right, which could be due to the vignette effects, flash effects or a combination of the two. Although it appears that the hamming and flattop windows are particularly susceptible to the spatial noise, it should be noted that the contrast in these images is over a much smaller range and therefore non-uniformities in Figure 14 (a) are not as visually apparent. As was shown in the thermal data previously, spatial non-uniformity can degrade defect identification since the standard deviation in the non-defective regions increases. Hence, the results in Figure 14 demonstrate that there is a need to apply corrections to the thermal data prior to the application of PPT. 


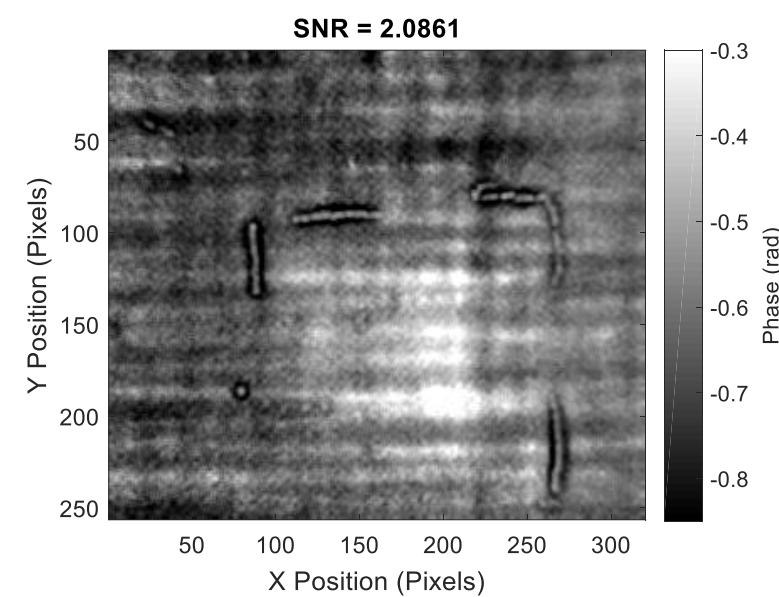

a) Rectangular Window

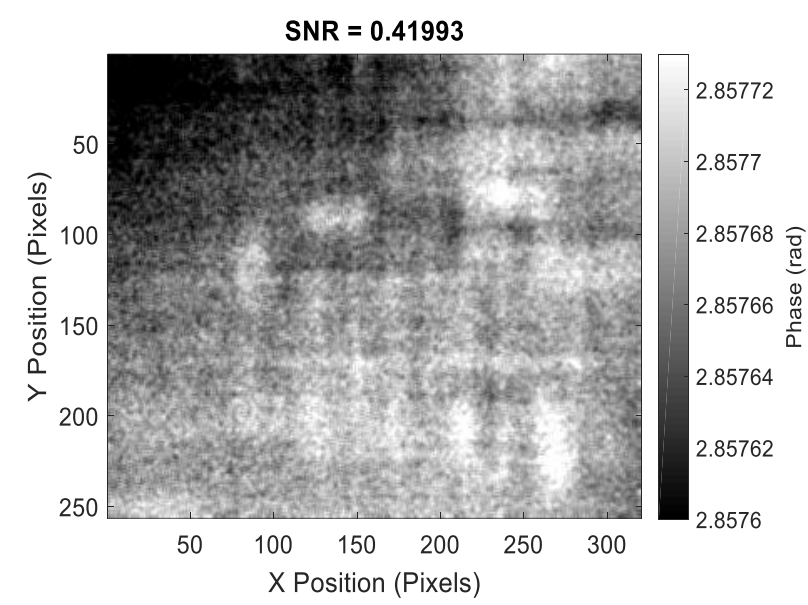

c) Flattop Window

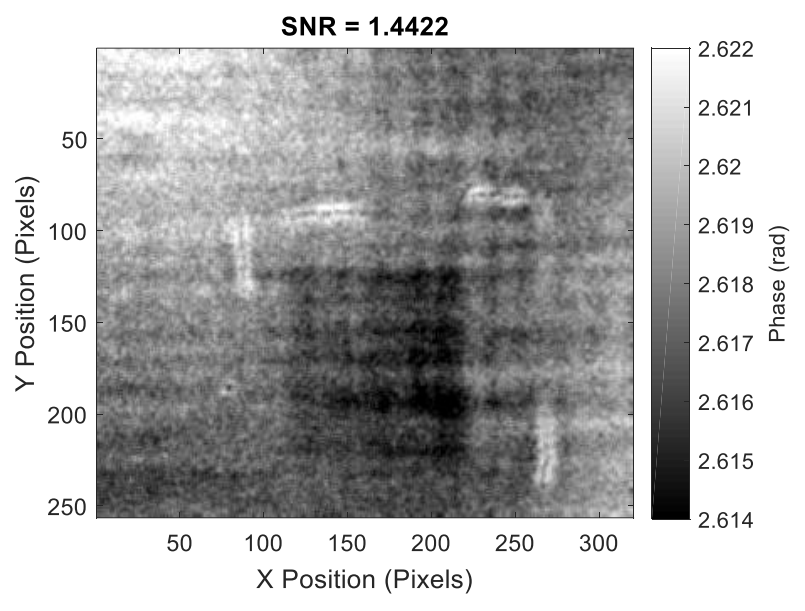

b) Hamming window

Figure 14: Comparison of three window functions applied to temporal thermal data from a PTFE insert at $1.8 \mathrm{~mm}$ depth. (Note: Lines around PTFE insert are pencil marks used for targeting IR detector.)

\subsection{Zero-padding}

To examine the effect of zero-padding and windowing, the three windowing functions were compared using raw thermal data from the PTFE insert placed at $1.8 \mathrm{~mm}$ depth. The data were then truncated to remove pre-flash frames $(N=2048)$ and then zero-padded to $N=8192$ samples before using the DFT. Phase images were selected based on SNR as described above in Section 7.2. Zero-padding resulted in the identification of the PTFE insert in a greater number of frequency bins for all windowing functions considered, as shown in Figure 15. When a rectangular window is applied (Figure 15 (a)), the phase contrast is low, and it is 
difficult to identify the defect because of the low SNR. In comparison with the images in Figure 14, the zero-padding provides improved identification when the hamming (Figure 15 (b)) and flattop windows (Figure 15 (c)) are used. However, the phase non-uniformity discussed previously can be seen in all the data, with flash effects and the vignette effect apparent in all three images in Figure 15, again indicating that an improvement in identification could be achieved by pre-processing the thermal data.

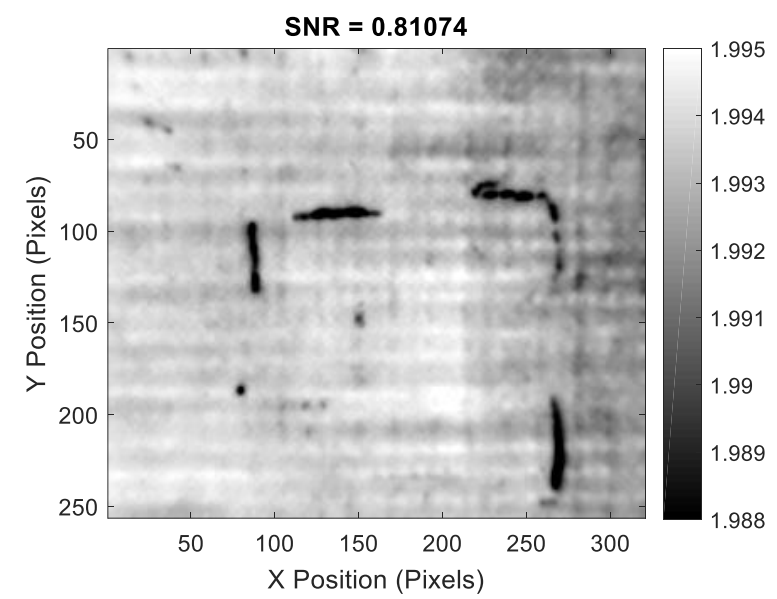

a) Rectangular Window

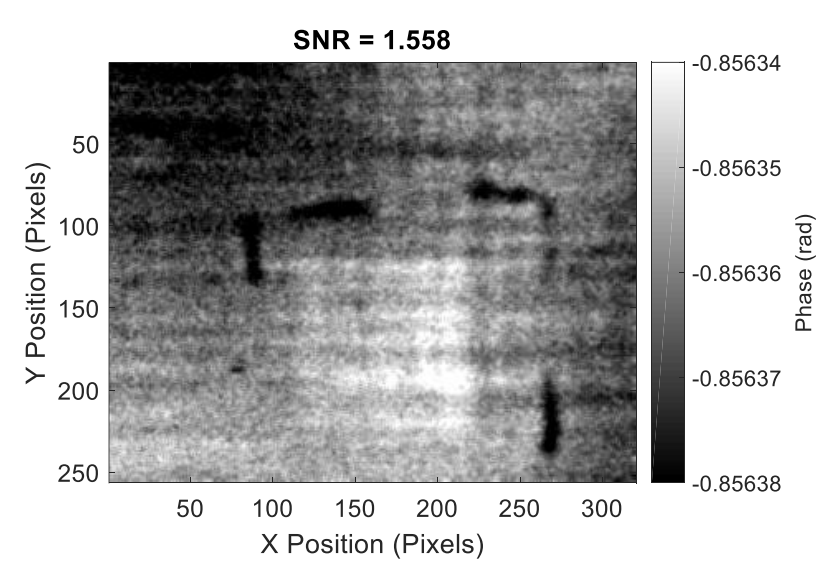

c) Flattop Window

Figure 15 - Comparison of three windowing functions with zero-padded from $\mathrm{N}=2048$ to $\mathrm{N}=8192$

using raw thermal data from a PTFE insert at $1.8 \mathrm{~mm}$ depth.

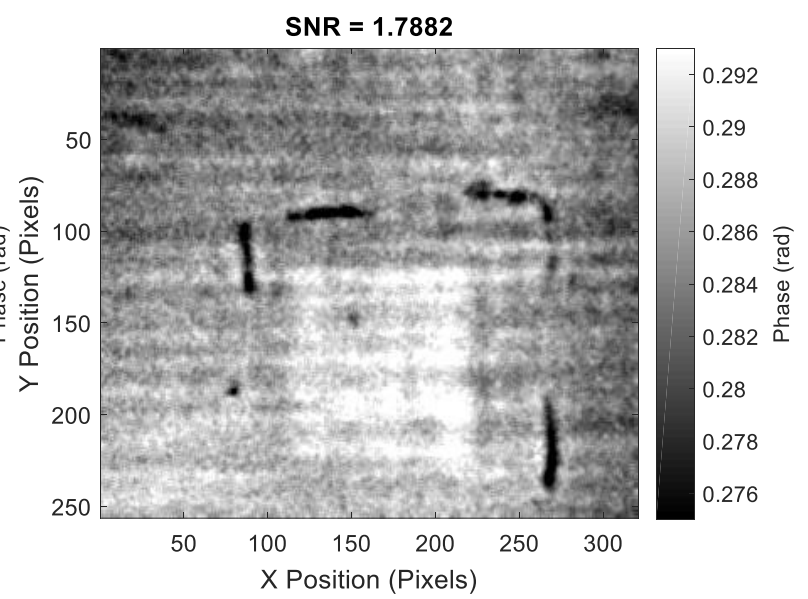

b) Hamming Window 


\subsection{Effect of Pre-Processing the Thermal Data}

Figure 16 shows phase data obtained by first correcting the thermal data as previously described, to reduce the effects of sensor cold spot, and non-uniform heating. TSR was then applied with a $6^{\text {th }}$ order polynomial, and PPT carried out using three windowing functions. The SNR was calculated for each frequency bin, and the phase images with the maximum ratio were chosen. Using the rectangular window the PTFE insert at $1.8 \mathrm{~mm}$ depth is still poorly characterised, and results in the lowest SNR of the three windows tested. In contrast, when the hamming and flattop windows are used the PTFE insert is clearly visible, and well characterised. There is little difference between the results obtain using hamming and flattop windows, although the flattop window provides the best SNR and identification of the square shaped PTFE insert. This indicates that for PPT applications, there is a low sensitivity to main lobe width. This is likely because PPT is a comparative technique, and the flat top window is applied to thermal decay signals measured at every pixel of the sensor array. The effect of the pre-processing of thermal data can be seen in all phase images, where the non-uniformities present in phase images shown in previous sections are minimised, and the phase noise has been significantly reduced by TSR. 


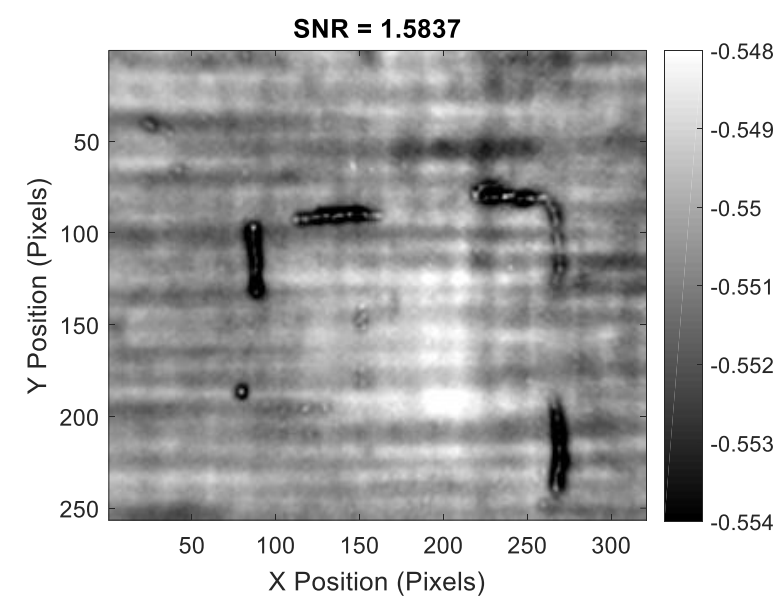

a) Rectangular Window

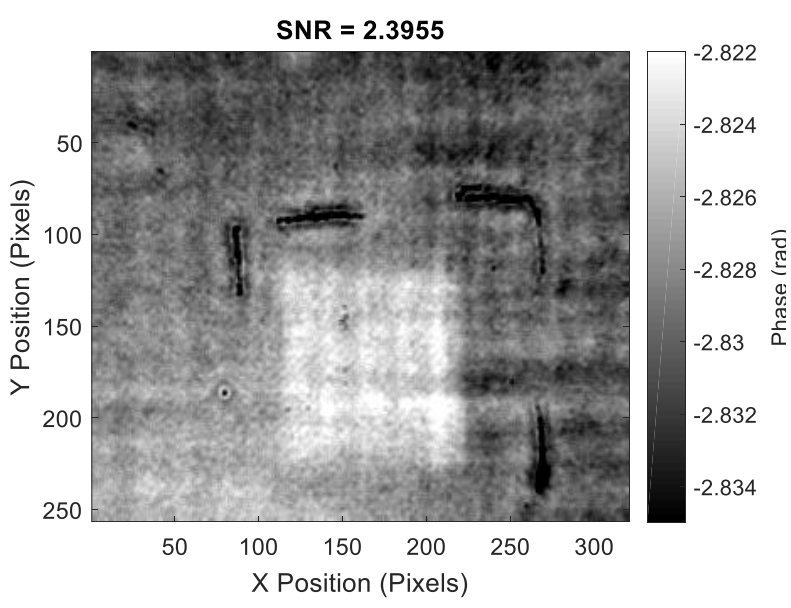

c) Flattop Window

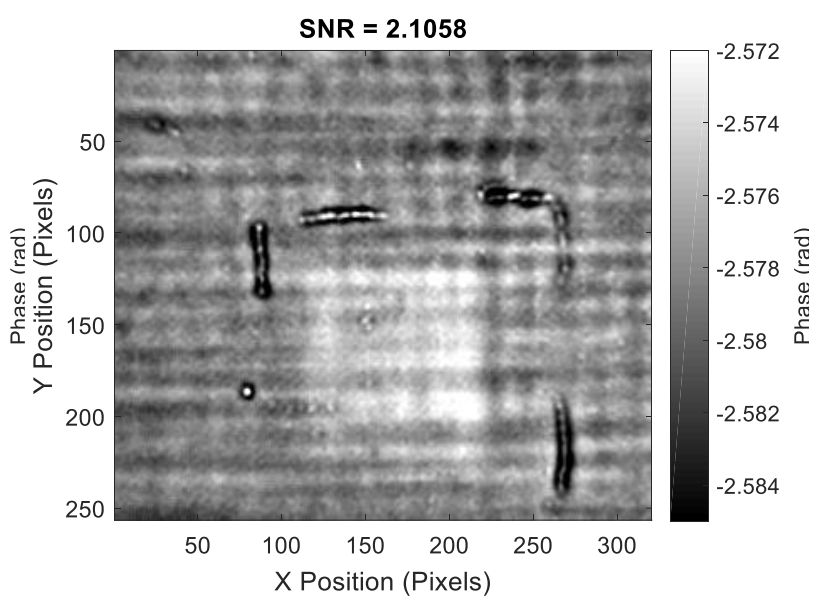

b) Hamming Window

Figure 16: Phase data obtained using vignette and flash compensation and TSR, showing comparison between three windows. Input thermal data truncated to remove pre-flash data, then zero-padded to $\mathrm{N}=8192$. Thermal data from PTFE inserts at $1.8 \mathrm{~mm}$ depth.

\subsection{Comparison of Windowing and Truncation}

As discussed previously, signal truncation to remove initial frames in the data set has been recommended when using PPT [20]. This has the effect of making a signal less transient and hence can improve phase contrast. To determine the optimum truncation for a specific inspection an iterative study must be performed, where the truncation is adjusted until the phase contrast is maximised. The hamming and flattop windowing functions also aim to make signal more stationary in nature, without requiring signal truncation. To determine the effect of 
differing truncations on the PPT data Figure 17 provides a comparison of the phase SNR with rectangular, hamming and flattop windows. The flattop and hamming windows almost always result in a higher SNR regardless of the truncation used when compared to the rectangular window, with the flattop window providing the best SNR of all when 700 frames are truncated. In addition, the SNR remains more stable when hamming or flattop windows are used, with only a marginal gain in SNR with increased truncation, until a peak at approximately 600-800 frames. The rectangular window appears to be more sensitive to truncation, with an increase in SNR of approximately $180 \%$ from no truncation to 800 frames. This implies that spectral leakage is important in PPT, because signal sampling has a significant effect on the phase SNR obtained.

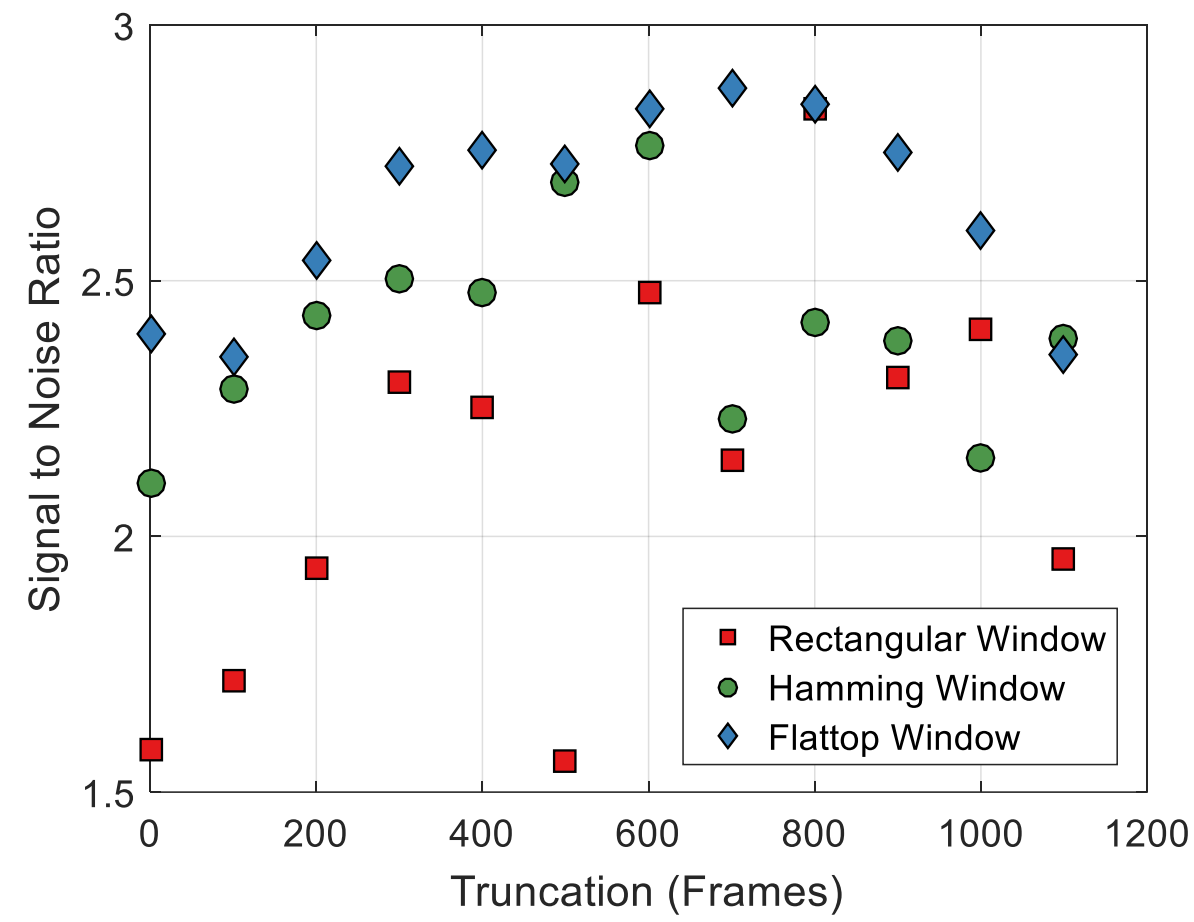

Figure 17: Effect of truncation on SNR of phase images using thermal data from PTFE insert at 1.8 mm depth. 


\section{Conclusions}

It has been demonstrated that by compensating for thermal non-uniformities, such as the vignette effect, and flash heating, significant improvements in thermal contrast are obtained in the time domain.

Corrections made to address random and systematic errors in the raw thermal data can significantly improve both thermal and phase contrast in PT and PPT inspections. Particularly, temporal smoothing was shown to reduce spatial noise in phase images due to the inherent coupling between temporal and frequency domains. This was most prominent in high frequency phase images, since TSR acts as a form of low pass filter. In PPT, the characterisation of defects typically improves with increasing frequency, smoothing therefore presents the greatest advantages for inspections of thin laminates. However, in thicker laminates where maximum probing depth is the primary objective and low frequencies are of greatest interest, the effects of temporal noise were observed, and it was shown to negatively affect SNR of phase images.

PPT is of greatest interest in cases where defects are not easily identified in the thermal data, and further processing is required if probing depth is to be improved. It was shown that simulated defects placed at $1.8 \mathrm{~mm}$ were not identified in thermal data, nor in phase images when conventional PPT was applied. The new approach of changing the windowing function to reduce spectral leakage improved the SNR, and enabled characterisation of the defects. Moreover, the best results were obtained when thermal corrections were combined with zeropadding and improved selection of the windowing function. It was shown that the flat top window, which results in highest side lobe attenuation and the least spectral leakage, performed best of the functions considered.

Signal truncation was shown to affect SNR in phase images regardless of the window used, however rectangular windows were much more sensitive to truncation when compared to hamming and flattop windows. It is shown that with no signal truncation, the flattop window performed the best in terms of SNR and characterisation. The flattop and hamming windows generally resulted in a higher SNR compared to the rectangular window, and the peak SNR was obtained using the flattop window. The work has demonstrated that implementing PPT 
with a flattop or hamming window represents an efficient alternative to the time consuming process of selecting optimal truncation parameters.

Although three popular windowing functions where chosen, it should be noted that numerous alternative functions exist, and this study is not exhaustive in this regard. It may however be concluded that the choice of windowing function can affect PPT phase data. In addition, it is in precisely the cases where PPT is most valuable, where thermal contrast is low, i.e. at deeper depths into the material, that the sensitivity to window selection is greatest. As such, it is important that the choice of window be reported alongside the PPT results since the omission of this information can restrict the reproducibility of work.

\section{Acknowledgements}

The authors would like to thank the Engineering and Physical Sciences Research Council (EPSRC) and BAE Systems Naval Ships for funding the work by an industrial CASE studentship. The work described in the paper was conducted in the Testing and Structures Research Laboratory (TSRL) at the University of Southampton and the authors are grateful for the support received from Dr Andy Robinson, the TSRL Experimental Officer.

\section{References}

[1] T. Dursun, C. Soutis, Recent developments in advanced aircraft aluminium alloys, Mater. Des. 56 (2014) 862-871. http://doi:10.1016/j.matdes.2013.12.002.

[2] W.J. Cantwell, J. Morton, The significance of damage and defects and their detection in composite materials: A review, J. Strain Anal. Eng. Des. 27 (1992) 29-42. http://doi:10.1243/03093247V271029.

[3] M.O.W. Richardson, M.J. Wisheart, Review of low-velocity impact properties of composite materials, Compos. Part A Appl. Sci. Manuf. 27 (1996) 1123-1131. http://doi:10.1016/1359-835X(96)00074-7.

[4] R.D. Adams, P. Cawley, A review of defect types and nondestructive testing 
techniques for composites and bonded joints, NDT Int. 21 (1988) 208-222. http://doi:10.1016/0308-9126(88)90333-1.

[5] D.P. Almond, S.G. Pickering, An analytical study of the pulsed thermography defect detection limit, J. Appl. Phys. 111 (2012) 093510. http://doi:10.1063/1.4704684.

[6] V.P. Vavilov, D.D. Burleigh, Review of pulsed thermal NDT: Physical principles, theory and data processing, NDT E Int. 73 (2015) 28-52. http://doi:10.1016/j.ndteint.2015.03.003.

[7] X. Maldague, S. Marinetti, Pulse phase infrared thermography, J. Appl. Phys. 79 (1996) 2694-2698. http://doi:10.1063/1.362662.

[8] C. Ibarra-Castanedo, N.P. Avdelidis, X.P. Maldague, Quantitative Pulsed Phase Thermography Applied to Steel Plates, Thermosense. 5782 (2005) 342-351. http://doi:10.1117/12.602360.

[9] D.P. Almond, S.L. Angioni, S.G. Pickering, Long pulse excitation thermographic nondestructive evaluation, NDT E Int. 87 (2017) 7-14. http://doi:10.1016/j.ndteint.2017.01.003.

[10] J.G. Sun, Analysis of Pulsed Thermography Methods for Defect Depth Prediction, J. Heat Transfer. 128 (2006) 329. http://doi:10.1115/1.2165211.

[11] S.G. Pickering, D.P. Almond, An evaluation of the performance of an uncooled microbolometer array infrared camera for transient thermography NDE, NDT E Int. 22 (2007) 63-70. http://doi:10.1080/10589750701446484.

[12] L. Sripragash, M. Sundaresan, Non-uniformity Correction and Sound Zone Detection in Pulse Thermographic Nondestructive Evaluation, NDT E Int. 87 (2017) 60-67. http://doi:10.1016/j.ndteint.2017.01.006.

[13] C. Ibarra-Castanedo, M. Genest, P. Servais, X.P. V. Maldague, a. Bendada, Qualitative and quantitative assessment of aerospace structures by pulsed thermography, Nondestruct. Test. Eval. 22 (2007) 199-215. http://doi:10.1080/10589750701448548.

[14] D.L. Balageas, J.M. Roche, Common tools for quantitative time-resolved pulse and step-heating thermography - Part I: Theoretical basis, Quant. Infrared Thermogr. J. 11 (2014) 43-56. http://doi:10.1080/17686733.2014.891324.

[15] S.M. Shepard, J.R. Lhota, B.A. Rubadeux, D. Wang, T. Ahmed, Reconstruction and enhancement of active thermographic image sequences, Opt. Eng. 42 (2003) 13371342. http://doi:10.1117/1.1566969.

[16] F. Galmiche, X. Maldague, S. Valler, J.-P. Couturier, Pulsed phased thermography 
with the wavelet transform, AIP Conf. Proc. 509 (2000) 609-616. http://doi:10.1063/1.1306105.

[17] J.W. Cooley, J.W. Tukey, An Algorithm for the Machine Calculation of Complex Fourier Series, Math. Comput. 19 (1965) 297-301. http://doi:10.2307/2003354.

[18] G. Busse, D. Wu, W. Karpen, Thermal wave imaging with phase sensitive modulated thermography, J. Appl. Phys. 71 (1992) 3962-3965. http://doi:10.1063/1.351366.

[19] C. Ibarra-Castanedo, X.P. Maldague, Review of pulse phase thermography, SPIE Sens. Technol. + Appl. 9485 (2015) 94850T. http://doi:10.1117/12.2181042.

[20] C. Ibarra-Castanedo, X.P.V. Maldague, Interactive methodology for optimized defect characterization by quantitative pulsed phase thermography, Res. Nondestruct. Eval. 16 (2005) 175-193. http://doi:10.1080/09349840500351846.

[21] E. Brigham, The Fast Fourier Transform and its Applications, Prentice-Hall, Eagle Woods, 1988.

[22] F.J. Harris, On the use of windows for harmonic analysis with the discrete Fourier transform, Proc. IEEE. 66 (1978) 51-83. http://doi:10.1109/PROC.1978.10837.

[23] D. Sundararajan, The discrete Fourier transform : theory, algorithms and applications, World Scientific Publishing Co. Pte. Ltd, 2001. http://doi:10.1142/4610.

[24] F. Weritz, Investigation of concrete structures with pulse phase thermography, Mater. Struct. 38 (2005) 843-849. http://doi:10.1617/14299.

[25] R.C. Waugh, J.M. Dulieu-Barton, S. Quinn, Modelling and evaluation of pulsed and pulse phase thermography through application of composite and metallic case studies, NDT E Int. 66 (2014) 52-66. http://doi:10.1016/j.ndteint.2014.04.002.

[26] V. Vavilov, Evaluating the efficiency of data processing algorithms in transient thermal NDT, (2004) 336. http://doi:10.1117/12.537604. 